\title{
Tangence
}

\section{Le Grand Livre magique des enfants non lecteurs}

\section{Anne-Marie Picard}

Numéro 54, mars 1997

Poétique du Livre

URI : https://id.erudit.org/iderudit/025939ar

DOI : https://doi.org/10.7202/025939ar

Aller au sommaire du numéro

Éditeur(s)

Tangence

ISSN

0226-9554 (imprimé)

1710-0305 (numérique)

Découvrir la revue

Citer cet article

Picard, A.-M. (1997). Le Grand Livre magique des enfants non lecteurs.

Tangence, (54), 101-119. https://doi.org/10.7202/025939ar d'utilisation que vous pouvez consulter en ligne.

https://apropos.erudit.org/fr/usagers/politique-dutilisation/ 


\section{Le Grand Livre magique des enfants non lecteurs ${ }^{1}$}

\section{Anne-Marie Picard}

... nous vivons plutôt ensommeillés afin de laisser l'Autre les yeux grands ouverts... ${ }^{2}$

\section{Le Gros Livre du Monsieur qui sait tout}

Catherine ${ }^{3}$ a dix ans et demi. Elle est venue du Portugal il y a quatre ans pour s'installer en France avec sa famille qui parle portugais "à la maison", c'est-à-dire dans l'intimité familiale. Catherine ne sait pas lire. Pourtant, dit sa mère, elle a *les forces pour apprendre mais elle est comme mon mari. Lui aussi, il a eu besoin d'aide à l'école. Puis elle ajoute: *Catherine est tout pour son père..... $M^{\text {me }} M$. a eu deux fausses couches: avant et après Catherine, des fausses couches restées secrètes. Lors des examens psychologiques, on remarquera chez Catherine une attention exagérée sur la présentation des objets à disposer: il faut que ce soit beau, parfait, bien aligné. Catherine veut être coiffeuse "comme ma mère, avant...". Lorsqu'on lui demande ce qu'elle attend, comment on peut l'aider, elle répond: "Je voudrais qu'on m'apprenne les mots que je sais pas». En effet, lorsqu'elle lit, elle. laisse carrément tomber ceux qu'elle ne reconnaît pas.

1 Il me faut dès le début de cette réflexion reconnaître ma dette envers les psychologues, psychanalystes et psychiatres de l'Unité de psychopathologie de l'enfant et de l'adolescent du Centre hospitalier Sainte-Anne à Paris, dont les travaux publiés et en cours me permettent de confronter ma pensée sur la lecture à la clinique et à son réel. Je tiens à remercier particulièrement Maïté Auzanneau de m'avoir permis l'accès à son travail d'écoute et de remédiation avec ses jeunes non lecteurs: sans elle cette réflexion n'aurait pu avoir lieu. Que celle-ci soit un hommage à son travail et à son éthique respectueuse du sujet.

2 Charles Melman, Noutelles êtudes sur l'bystérie, Paris, Joseph Clims, coll *Le Discours psychanalytique *, 1984, p. 126.

3 Les noms ont été changés pour préserver l'anonymat. Tous les exemples de cas sont tirés des consultations de Maité Auzanneau ou du $D^{r}$ Lionel Bailly de l'Unité de psychopathologie de l'enfant de Sainte-Anne. 

Catherine?

- Comment est-ce que tu penses que l'on apprend à lire,

- Un monsieur avait appris tous les mots à mon père. Il les avait appris dans un gros livre d'histoire.

- Et comment fait-on pour trouver ce livre?

- 'Chais pas.

Acquérir ce livre et le savoir qu'il symbolise n'intéresse pas Catherine. De la même façon qu'elle ne sait pas si elle veut vivre en France ou au Portugal. C'est justement cette attitude vis-à-vis du savoir, étayée par un imaginaire qui "l'arrange", qui entrave les performances cognitives de la petite Catherine. Cette situation psychique qui noue une théorie erronée de la lecture à un nonvouloir-savoir, la première excusant la seconde - ce n'est pas moi (qui ne veut pas), c'est l'Autre (qui sait) - , se retrouve chez d'autres enfants en graves difficultés dites "scolaires". Il y a, par exemple, David, qui raconte que: "C'étaient les cigognes qui avaient apporté les livres à maman", à sa maman qui était... bibliothécaire. Où est-il, lui, dans ces rangées de livres qui occupent sa mère? De quel désir procède-t-il? Quel risque encourraitil de le savoir?

Carol Rose, dans Des enfants hors du lire, propose une typologie de ces théories erronées ${ }^{4}$ que se donnent les enfants ${ }^{5}$, fina-

4 Carol Rose, -Lecture, croyances, doute et fragilité. Le "dilemme existentiel" des enfants non lecteurs:, Des enfants bors du lire. Sous la direction de Christiane Préneron, Claire Meljac et Serge Netchine, Paris, Bayard/INSERM CTNERHI, coll. “ Païdos/Recherche*, 1994, p. 177-190.

5 Donnons ici les critères stricts établis par l'équipe de Sainte-Anne pour définir le groupe des - non-lecteurs *: ces enfants doivent être "âgés de neuf ans au moins au moment du premier contact, doivent avoir accompli leur scolarité en français à partir de la maternelle-, ne doivent pas présenter de *troubles psychopathologiques invalidants tels que psychoses, ni de troubles évidents du langage de type dysphasie, ni de signe patent d'atteinte du système nerveux central *, ils doivent "faire preuve d'aptitudes normales à l'une des deux échelles du WISC-R* (test du QI qui doit, chez eux, avoir des résultats égal ou supérieur à 85 à l'échelle verbale ou en perfornance); ils ne doivent pass. appartenir à une famille particulièrement défavorisée ou visiblement pathogène *; et enfin, ils doivent être "gravement infériorisés dans leurs activités de lecture : les enfants retenus dans la recherche ne -dépassent pas, à neuf ans et au-delà, un niveau d'acquisition présent, habituellement, dès la première année de scolarité *. Leur déchiffrement d'un texte est lent et pénible et ne permet pas de se dégager du texte. Les cas de lecture totalement impossibles 
lement, "pour ne pas lire". Nous pouvons citer quelques-unes de ces croyances compactes qui sont de plusieurs ordres, par exemple: r lire, ce n'est pas produire du sens mais c'est avant tout une activité imposée par autrui, en général par l'adulte. (p. 179); ou bien: "lire, c'est apprendre par cour" (p. 179); ou encore: "lire, c'est nommer des lettres" (p. 180), etc. Ces croyances sont difficilement questionnables (souvent induites par l'autorité pédagogique, parent ou "maitresse" bien intentionnés; voir Rose, p. 179) car elles sont * utiles * pour l'enfant, psychiquement, narcissiquement : elles lui permettent d'abord de ne pas être responsable de ses échecs - échecs souvent "recherchés" - puis de ne pas laisser de place au doute - un doute qui ne pourrait s'appuyer que sur l'hypothétique et donc sur une liberté de penser et d'exister qui est, pour l'instant, impossible pour ces petits apprenants (voir Rose, p. 178).

Ce qui nous arrêtera ici est une "théorie" non discutée par l'auteur, celle qui se fonde sur la croyance en l'existence d'un "Grand Livre" quelque part, où sont écrits tous les mots et qui est détenu par un autre, lointain. Il nous semble que cette croyance subsume les autres, qu'elle se situe en amont des autres. Elle constitue en effet une véritable théorie du langage, du symbolique et c'est en tant que telle qu'elle travaille les autres croyances relevées par Carol Rose. Les cigognes de David ou le Monsieur de Catherine qui enseigne avec un gros livre sont des figures de l'omniscience: oiseaux, messagers des dieux, qui voyagent entre ciel et terre pour apporter les livres de la maman (et les bébés?); vieil homme un peu mage, étrange et inaccessible, qui détient les clés du savoir du père, lui-même "enfant en difficulté ", un Monsieur savant qui réside au Portugal, lieu de l'enfance perdue. Pour Catherine et pour David, il faut ainsi en passer par l'omniscience pour obtenir la "petite science. que représente la lecture comme premier stade pressenti du reste de l'acquisition des connaissances. Pour l'instant, il est "bon " pour eux de garder le savoir dans l'Autre et ils ne sont aucunement intéressés par l'exercice qui consisterait à se l'approprier. C'est qu'ils vivent dans le monde magique de la langue maternelle, paradis de l'inceste imaginaire

font "évidemment partie de [la] population " sélectionnée. S. Netchine, $\mathrm{Cl}$. Meljac, Ch. Préneron, "Introduction: non-lecteurs dans un univers de signes. Le sens d'un échec *, Des enfants bors du lire, op. cit., p. 11-25 et p. 19-20. 
où ils sont "tout pour papa" ou a tout pour maman". Que vouloir d'autre, alors, que le statu quo? Qu'ont-ils à gagner d'être curieux?

\section{Théories erronées et croyances : comment ne pas accéder au savoir}

Rappelons comment Freud nous raconte les effets de la poussée de savoir (Wissensdrang) et comment l'enfant en vient à un arrêt de la curiosité, arrêt qui, fondé sur une théorie sexuelle erronée devenue système, fera fond d'une croyance qui permet de ne pas déloger le sujet comme phallus imaginaire de la Mère.

Il me semble découler de nombreuses informations que les enfants refusent de croire à la théorie de la cigogne, mais après avoir été ainsi une première fois trompés et repoussés, ils en viennent à soupçonner qu'il y a quelque chose d'interdit que les a grandes personnes - gardent pour elles et, pour cette raison, ils enveloppent de secret leurs recherches ultérieures. Mais ils ont aussi vécu par là la première occasion d'un *conflit psychique dans la mesure où des opinions, pour lesquelles ils éprouvent une préférence de nature pulsionnelle mais qui ne sont pas *bien* aux yeux des grandes personnes, entrent en opposition avec d'autres, qui sont fondées sur l'autorité des grandes "personnes", mais qui ne leur conviennent pas à eux. Ce conflit psychique peut devenir bientôt un *clivage psychique"; l'une des deux opinions, qui va de pair avec le fait d'être $u n$ bon petit garçon mais aussi avec l'arrêt de la réflexion devient l'opinion dominante; l'autre, ayant reçu, entre temps, de la part du travail de recherche, de nouvelles preuves qui n'ont pas le droit de compter, devient l'opinion réprimée, • inconsciente . Le complexe nucléaire de la névrose se trouve constitué par cette voie. (G.W. VII, $\$ 176$, nous soulignons ${ }^{6}$ )

[...] les enfants produisent beaucoup de choses fausses dans le but de contredire une connaissance plus ancienne, meilleure mais devenue inconsciente et refoulée.... Chez beaucoup d'entre eux, le refoulement sexuel s'est développé si avant qu'ils ne veulent rien entendre, et ils réussissent à demeurer ignorants même dans les années ultérieures, apparemment du moins jusqu'à ce que dans la psychanalyse des névrosés le savoir émanant de la première enfance vienne à jour. Je connais aussi deux garçons qui ont entre dix et treize ans et qui certes ont

6 Traduction dirigée par J.-B. Pontalis, document ronéotypé, C.H. Sainte-Anne. 
reçu des explications sexuelles, mais ont opposé à celui qui s'en portait garant cette fin de non-recenoir: il se peut que ton père et d'autres se comportent de la sorte, mais je suis bien sâr que mon père, lui, ne ferait jamais ça. ( $\$ 187-88$, nous soulignons)

Rester un bon petit garçon se fait au prix de la curiosité et de la vérité. L'enfant se soumet à "l'opinion des grandes personnes" et évite ainsi la punition possible. Sa découverte, chargée de jouissance interdite, est alors réprimée. Le savoir qu'il pourrait acquérir par lui-même ne compte pas: il cesse de chercher, car trouver c'est courir le risque d'insatisfaire les parents. Ce qu'il pourrait en effet trouver, c'est le désir, le manque et donc la castration des parents en tant qu'ils sont eux-mêmes soumis à l'Autre. Il s'en trouvera par là même évincé de la scène primitive comme phallus tout satisfaisant puisque ce dernier s'en trouve rejeté à la place de l'Autre, en amont de la jouissance, comme cet Un divin qui habite le corps des parents le temps de leur rapport sexuel. Ainsi, pour ces deux garçons dont parle Freud, qui posent leur père comme exception (tous mais pas lui): si le père n'est pas commandé par le désir de l'Autre, il reste omniscient, "toutjouissant " comme le père primitif de la horde avant le meurtre et le refoulement de S1, induit par cet Autre, ne pourra se faire: la métaphore (qui est chute de S1 sous la barre) demeurera alors impossible. De là découlera l'absence de jeu dans la langue, l'absence de case vide, et donc l'élaboration symbolique: comme dans le jeu du taquin où c'est la case vide qui permet aux autres pièces de bouger pour faire figurer une image ou pour énoncer un mot ou une phrase.

Rester un bon petit garçon signifie ne pas se détacher, surtout ne pas dépasser ce sentiment d'être laissé pour compte dans et par le désir des parents, ceci évitant - et c'est là le bénéfice de l'arrêt de la pulsion de savoir - le sentiment angoissant d'être étranger ${ }^{7}$ à ses parents. Les théories erronées des enfants ${ }^{8}$ leur

7 Voir Sigmund Freud, "Le roman familial des névrosés", Néursse, psychose et periersion, Paris, PUF, 1974, p. 158.

8 -Avec la découverte que l'enfant se développe dans le corps de la mère, découverte qu'il fait encore indépendamment, l'enfant serait sur la bonne vole pour résoudre le problème sur lequel il met d'abord à l'épreuve la force cle sa pensée. Mais il est inhibé dans la suite de ses progrès par une ignorance, que rien ne peut pallier et par de fausses théories que l'état de sa propre sexualité lui impose. [...] Ces fausses théories sexuelles [...] ont toutes une propriété remarquable. Bien qu'elles se fourvoient de facon grotesque, 
106

permettent de ne pas penser plus avant: ils se concentrent ainsi sur le comment (on fait les enfants) au lieu de continuer à se poser la question du pourquoi (mes parents m'ont fait), question qui articule celle de leur désir et finalement du sens de l'existence?. Pour certains d'entre eux, peut-être est-il moins "dangereux" de ne pas savoir. Cependant, éviter cette confrontation et cette traversée d'un savoir interdit parce que signifiant le manque (à satisfaire) aura pour conséquence l'inhibition de la pulsion de savoir et l'impossibilité d'advenir au désir, à l'hypothèse de l'existence de l'objet et donc à la sublimation.

Les enfants en grande difficulté de lecture, nous dit Maïté Auzanneau, "fonctionne[nt] dans le "tout va bien", "tout doit aller coûte que coûte" ". Après des années d'échec où "au problème spécifique de lecture s'ajoutent la honte, la culpabilité et une tendance à la dissimulation n, ces enfants sont parvenus à développer

des stratégies inadéquates, s'ingénient à un maximum de solutions, afin de fabriquer une carapace dans laquelle ils se trouvent, tout compte fait, relativement bien*: dépendance à la mère, à lautre en général et, plus tard, statut reconnu leur donnant droit à une aide sociale d'Etat et/ou organisme de formation. Tout est fait pour déclencher le rejet et l'insuccès [... . . ${ }^{10}$

Les croyances de ces enfants sont des exemples flagrants de ce que Joyce McDougall appelle des "solutions oniriques", solutions qui sont apportées à un "désir de l'impossible. Impossible car ce que cherche paradoxalement ce désir, c'est le statu quo, l'éternisation de la scène de l'imaginaire (incestueux) où se déroule leur théâtre privé. Ce désir de l'impossible traverse toutes les mises en scènes humaines, toutes les croyances. Car "[clomment l'être humain peut-il [...] affronter ces échecs scanda-

chacune d'elles contient pourtant un fragment de pure ikerité. [...] Ce qu'il y a en elles de correct et de pertinent s'explique par le fait qu'elles trouvent leur origine dans les composantes de la pulsion sexuelle [nous soulignons] qui sont déjà à l'ouuvre dans l'organisme de l'enfant; ce n'est pas l'arbitraire d'une clécision psychique ou le hasard des impressions qui ont fait naître de telles hypothèses, mais les nécessités de la constitution psycho-sexuelle, et c'est pourquoi nous pouvons parler de théories sexuelles infantiles typiques, c'est aussi pourquoi nous trouvons les mêmes conceptions erronées chez tous les enfants dont la vie sexuelle nous est accessible. (traduction dirigée par J.-B. Pontalis, \$177).

9 Voir Marc-Léopold Lévy, "La cigogne et le kabbaliste ou du comment et du pourquoi ^, Texte, L'imaginaire de la théorie, $\mathrm{n}^{\mathrm{os}}$ 17-18, 1996, p. 233-242.

10 Maïté Auzanneau, - Que faire pour sortir les non-lecteurs de leur état de nonlecteur? ", Des enfants hors du lire, op. cit., p. 376. 
leux du narcissisme que sont l'altérité, la différence des sexes, le vieillissement et la mort, autrement que par la pensée magique qui, fantasmatiquement, le transforme en être tout-puissant, bisexué et immortel?:11; c'est-à-dire objet tout-satisfaisant pour la mère ou pour le père et donc non advenu comme sujet de son désir propre. Cet état des choses et des relations que les solutions oniriques cherchent à préserver, afin finalement d'éviter la division et la séparation, a pour corrolaire mythique ce que la chrétienté entend par ale paradis d'avant la chute". Si les croyances de ces enfants sont - rigides et cloisonnées " ${ }^{12}$, qu'elles font "bloc", c'est que, comme toutes croyances, elles permettent au sujet qui y fait appel de "gagner son ciel". Un ciel atteint au prix du désir (de savoir) et donc au prix de la créativité et de la participation dans l'aire culturelle. Ce sont les fondements psychiques de cette "pensée magique " et de ce "paradis" qui vont nous arrêter maintenant.

\section{Pensée magique, ostension et exophore : Au commencement était la jouissance... incestueuse}

Un jour, on reconnaît le mot sur la page, on le dit à voix haute et c'est un bout de Dieu qui s'en va, une première fracture du paradis...

Christian Bobin, Une petite robe de fête

Si nous reprenons, un peu tronquée, l'exergue de Christian Bobin que les auteurs des Enfants bors du lire ont aussi choisie ${ }^{13}$, c'est qu'il nous faudra bien parler de ce Dieu-là, d'un dieu un peu plus abstrait que celui de l'écrivain, catholique fervent, puisqu'il sera pour nous le représentant de l'Un, du mythe de l'unité perdue $^{14}$ du sujet. Il se tient en un temps 0 et tient lieu d'une jouissance illimitée. Il est là où résiderait (au mode conditionnel) le sujet humain dans ce temps mythique d'avant la Chute, c'est-à-dire

11 Joyce McDougall, Théâtres du Je, Paris, Gallimard, 1982, p. 12.

12 Carol Rose, op. cit., p. 178.

13 Pour illuminer par la poésie de Bobin l'ouverture du compte rendu collectif des résultats de quelque huit années de recherche de l'Unité de psychopathologie mentionnée ci-dessus.

14 Nous sommes fidèle en cela à la conception que s'en fait Marc-Léopold Lévy dans - De l'Origine de Dieu , Cbe Vuot? Revue de psychanalyse (à parâtre). 
108

avant son rejet hors d'un monde où ses besoins étaient satisfaits- - sans parole ni demande. Cette chute, préliminaire à toute vie, à toute humanité, est "la fracture" dont parle Bobin. Elle métaphorise une perte irrémédiable. Qu'est-ce que l'humain perd du fait même qu'il est humain et qu'il parle? Il perd un monde, celui de son animalité, de l'instinct réglé par l'ordre naturel. Il perd la possibilité d'être comblé. Ce paradis, c'est bien l'invention nostalgique d'un espace-temps où toute pulsion serait coextensive de sa satisfaction complète, et l'humain un animal muet, un chat satisfait et ronronnant. Dieu est la représentation de ce qui manque à l'humain du fait qu'il parle, du fait qu'il a à en passer par le symbolique pour demander ce qu'il veut, et qu'il est donc aliéné au langage et à la volonté de l'autre. Au paradis, Dieu devine les besoins. Il est un hôte hospitalier, une bonne mère qui pourvoit à tout. Être Dieu, c'est avoir le pouvoir de faire apparaitre l'objet, comme la bonne marraine des contes:

Sa marraine qui la vit toute en pleurs, lui demanda ce quelle avait. Je voudrais bien... je voudrais bien... I... Sa marraine, qui etait fée, lui clit: Tu voudrais bien aller au bal, n'est-ce pas? [...] Hé bien... je t'y.ferai aller. Va dans le jardin et apporte-moi une citrouille. Sa marraine (... J la frappa de sa baguette, et la citrouille fut aussitôt changée en un beau carosse tout doré [...]. ${ }^{15}$

Dans le monde de la magie, il y deux solutions pour faire apparaître l'objet: ou les fées pointent le réel avec leur baguette, ou elles énoncent une formule compliquée et secrète.

Les contes mettent en scène l'impossible comme ce qui est rendu possible par le pouvoir nominaliste des fées. Leur magie transforme en effet le gouffre infranchissable entre signifiant et référent en une relation de clésignation "rigicle":

1) avec la baguette, une chose est montrée dans la cosmogonie banale et indifférente du quotidien (citrouille, rats, lézards, haillons), qui se métamorphose - non pas en un objet partiel (ce qui serait symbolique) - mais en l'objet définitif car il comble totalement les rêves "les plus fous et les plus impossibles": il est toujours le plus beau que l'on ait jamais vu - l'impossible se marquant ici par le subjonctif. Par exemple, les vilains habits de Cendrillon se transforment en "des habits de drap d'or et d'argent tout chamarrés de pierreries", qui deviendront "les grandes beau-

15 Charles Perratult, "Cendrillon •, Contes, Paris, Flammarion, [1989] 1991, p. 279. 
tés de cette inconnue [...] si belle et si aimable" (Perrault, 280), admirée de tous, enviée de toutes. La baguette empêche le glissement de sens, prévient toute ambiguité en touchant la chose qui deviendra l'objet désiré: comme représentation matérielle de l'ostention, elle pointe, découpe un segment du réel (dans le sens banal du terme) et le transforme en l'objet même de la jouissance. Il s'agit d'une monstration unidirectionnelle et donc univoque: personne d'autre que la bonne fée ne peut utiliser ni la formule ni la baguette sous peine de danger;

2) les formules magiques sont idiosyncrasiques, discrètes et incompréhensibles pour tout autre: elles appartiennent en propre à leur énonciatrice, il en faut une différente pour chaque situation, elles ne servent qu'à faire une chose seulement. D'où l'exigence de ce "Grand Livre" de magie qui apparaît souvent dans les légendes, une encyclopédie imaginaire qui contiendrait toutes les formules et tous les objets désirables possibles, objets comptabilisés et attachés à la formule unique de leur demande.

Le langage magique ne connaît pas les déictiques (on ne dit nullement: "Apporte-moi cette citrouille-là"): il ne renvoie pas au sujet énonciateur mais, au contraire, locculte. Le mettre en jeu serait le constituer comme manquant et le soumettre à la formulation de sa demande et donc au désir de l'Autre (à la capacité de ce dernier d'entendre et de comprendre, et de vouloir répondre). Nous serions alors déjà entré dans le monde chaotique du désir sous la tutelle de l'Autre. Puisque, dans, le monde magique, le langage nomme et saisit toujours son objet - il n'est pas un discours - il n'y a pas de place pour le doute ou l'ambiguité: il y a une place pour chaque chose et chaque chose est à sa place. En effet, la marraine, et le Dieu du paradis, représentent, en lui clonnant figure, consistance, le rêve d'un monde où le désir deviendrait réalité, c'est-à-dire un monde "qui marcherait", où tout coünciderait, où, finalement et en termes lacaniens, "il y aurait du rapport sexuel", un mode non entamé, sans jeu, sans case vide, sans impossible - et l'objet donné y serait d'ailleurs toujours un objet phallique. Dans ce monde (qui est pour M.-L. Lévy celui de la langue dite maternelle, avant que le père, dans le meilleur des cas, n'y instaure du jeu, de la transgression en s'y représentant comme désirant ${ }^{16}$ ), le "sujet " ne serait donc pas aliéné au

16 -La Langue maternelle, conférence donnée à L'École des hautes études en sciences sociales de Paris, avril 1996. 
signifiant, au ratage inéluctable de la désignation puisqu'un signifiant n'y renverrait pas à un autre signifiant mais à la chose même, le sujet atteignant toujours son but, et finalement ne désirant pas.

La geste créationniste de Dieu et le geste prestidigitateur de la fée présupposent une théorie du langage où les signaux émis le sont dans une parfaite correspondance entre énonciation et énoncé, entre signe et réalité. Un langage sans autonomie, sans existence hors de la situation de son émission (comme celui des animaux) puisqu'il ne peut faire référence qu'à ce qui est immédiatement visible, sensible. La formule magique (comme la langue de Dieu) est sans cases vides, sans déictiques ni anaphores. Elle fonctionne pour métamorphoser les choses en objets phalliques ou, pour le dire autrement, pour transformer les indéfinis en exophores - exophore que l'on retrouve, par exemple, dans "le" soleil où le déterminant de l'universel présente le référent comme connu par tous, en dehors donc de la situation d'énonciation et du discours ${ }^{17}$ ). Le pointage de la baguette accomplit une opération qui transforme une citrouille en quelque chose qui prend une valeur reconnaissable par tous: le plus beau carrosse. Le particulier, pointé, acquiert un statut universel, "reconnu". La métamorphose opère ainsi un découpage et une érection véritablement phallique: la chose se dresse en phallus/ désirable dans le lieu commun de la valeur sociale.

Catherine Ferron et les co-auteurs d'une recherche sur les conduites narratives des non-lecteurs remarquent ${ }^{18}$ la tendance importante qu'ont ces enfants à utiliser ces formes exophoriques. Ils notent ainsi la grande indétermination référentielle dans leurs paraphrases de récits: les héros, même inconnus par le grand public, ne sont pas présentés, l'antécédent des pronoms est présupposé, etc. Ces présuppositions impliquent une croyance très forte: celle que le savoir est partagé par tous et donc qu'il y a "la possibilité pour le destinataire de reconstituer ou identifier la réa-

17 Voir G. Kleiber, * Déictiques, embrayeurs, comment les définir? *, L information grammaticale, $\mathrm{n}^{\prime} 30,1986$, p. 3-22.

18 Catherine Ferron, - Enfants non lecteurs en thérapie "Penpectives pychiatriques, vol. IV, $\mathrm{n}^{\circ} 24,1990$, p. 262-265; et "Qu'est-ce que c'est que ça?", Quand lalangue fait symptôme. La psychanalyse de l'enfunt. $\mathrm{n}^{\circ}$ 3/4, 1987. p. 105-124; et Christiane Préneron, Anne Salazar-Ortiz, Marie KuglerLambert, Jacques David, "Les conduites narratives: récits d'enfants non lecteurs, " Les enfunts bors du lire, p. 209. 
lité dont [l'enfant] parle"19. Ce qui est partagé, pourrions-nous ajouter, c'est bien le référent comme Un, sa possibilité objective, son indivisibilité: il est cet objet recherché dans le regard même de l'interlocutrice, fée plus ou moins bonne qui, en parlant, fait surgir du néant des mots-choses parfois reconnus, parfois monstrueux: Mais de quoi parle-t-elle? Que (me) veut-elle par sa question et son doigt tendu? KESKESEXA ${ }^{20}$ ? "La coordination indicée manque aux enfants non lecteurs " constate Ferron, "le sujet ne l'énonciation ne parle que par son absence" ${ }^{21}$.

Le langage dans la séance est minimum, à l"emporte-pièce, holophrastique: "Voilà, voilà *. Ces enfants nont rien à dire, à raconter. Où trouver pour le thérapeute quelque chose susceptible d'accrocher, de soutenir un désir?

Création de signifiant et holophrase sont les cleux faces d'un même manque. [...] Leurs voix semblent ne pas avoir de support corporel; leur regard se perd souvent clans un ailleurs maternel dont ils ne se cléfendent que par le symptôme: ils sont sujet clu rêve cle la mère [...]. ${ }^{22}$

La pensée interdite de ces enfants fait symptôme: inhibition, manque d'autonomie, troubles de la concentration, rigidité, fuite de la situation et répétitions stéréotypées ${ }^{23}$ mais aussi agitation ou apathie, absence de curiosité, angoisses où insiste la culpabilité que le regard social, institutionnel, jouant comme tiers a sans doute "réussi" à créer faute souvent d'un père qui ne se pose un peu là dans le discours de la mère.

\section{Deixis, métaphore paternelle et hypothétique : puis vint la chute}

Si c'est Dieu (et ses représentants) qui a tout inventé, alors il sait tout. Un jumeau, Nicolas, découpe les lettres deux par deux pour les cléchiffrer, mais, parfois, il doit s'arrêter, sidéré, devant un mot.

- "Pourquoi as-tu peur, Nicolas?

19 Catherine Ferron, * Enfants non lecteurs en thérapiè, op. cit., p. 208.

20. Idem, "Qu'est-ce que c'est que ça?", p. 111.

21 Idem, "Enfants non lecteurs en thérapie", op. cit., p. 262

22 Ibid., p. 264.

23 Voir Mäté Auzanneau, "Que faire pour sortir les non-lecteurs de leur état de non-lecteur?", op. cit., p. 377. 
112

- Je ne connais pas le mot parce que ce n'est pas moi qui l'ai inventén.

Seul celui qui l'a inventé peut donc le lire. Face à la page du livre de lecture, des enfants tels que Nicolas devinent au lieu de déchiffrer. C'est qu'ils font appel à la divination de leur mère qui sait tout et qui n'a rien interdit. Ne pas accéder au savoir, c'est pour mieux le laisser à leur mère et rester tout pour elle.

- Quelle est ta date de naissance? demande-t-on à Valérie. fant.

- Je ne sais pas. Ma mère ne me l'a jamais dit, répond l'en-

- Comment lit-on? demande-t-on à la mère.

- Il faut lire bien tout, répond-elle. On met deux lettres ensemble...

Nicolas et Valérie gardent le savoir dans l'Autre. Pour ne pas perdre, ne pas se perdre de vue comme *tout présent pour la mère "ou pour le père (ou tout autre pouvant être tenu par l'enfant à cette place du tout-savoir). Dieu, la marraine, le magicien sont des figures de cette omniscience dans nos légendes et nos mythes. Ils possèdent tous la science innée quant aux désirs de leurs "sujets". Mais ils savent aussi ce qui est bien pour eux: ils connaissent en effet la Loi, une Loi qui les transcende, sur laquelle ils n'ont pas de pouvoir. Cette Loi apparaît afin de limiter, borner le possible appétit ogresque du héros pour une jouissance éternelle qui serait mortifère. Cette limite instaure le temps, invente l'Histoire en nouant le symbolique à cet imaginaire atemporel et figé autour d'un prétendu objet présentifiable. Pour éviter qu'il ne soit aveuglé par cet objet (le plus beau qu'on ait jamais vu...) ou que l' assouvissement total de tous ses voeux n'aboutisse à la mort du héros, on ne lui en accordera souvent que trois. Il doit alors utiliser son intelligence, faire des hypothèses. Quant à la marraine de Cendrillon, la limite temporelle qu'elle impose fait office de principe de réalité : passé minuit, l'objet redeviendra bout de réel et donc, plus de jouissance possible! Quant à la jouissance paradisiaque de la Bible, elle se trouve littéralement marquée par une borne: Dieu la pose au centre du Jardin par un geste ostensif qui fonde la proton deixis - car auparavant nul besoin de montrer, de découper le réel pour le connaître. De cet arbre-là, vous ne mangerez pas!: mais pourquoi donc tout sauf cet arbre-là ? On sait que c'est 
cette restriction même qui "permettra" la chute, c'est-à-dire l'advenue des deux "êtres" du paradis à l'humanité. Comment cela fonctionne-t-il? En disant Tout sauf ça, que fait Dieu? Qu'est-ce que c'est donc que "ça"?

Le Tout sauf ça de Dieu rappelle une autre interdiction, celle de la mère à son enfant qui va commettre une bêtise: Tout... mais pas ça! dit-elle. Comme pour Dieu, sa parole fait acte: elle établit une restriction, installe une *xclusion. qui en fin de compte instaure un vide dans ce qui, autrement, est un espace régi par sa toute-puissance, comme nous le rappelle Bergès ${ }^{24}$, un espace où tout est à sa place. Quel vide s'instaure ici de ce sauf ça, de cette proton deixis? Le vide créé est celui de l'objet de désir, et le premier objet, c'est bien l'objet de désir de la mère. C'est de l'émergence de la métaphore paternelle dans le discours de celle-ci qu'il s'agit ici: "Tout, nous dit Bergès, c'est la mère, en somme l'unité, la globalité: tout est prévu, tout est là ... mais pas ça!, c'est le père" (p. 261). Ce qui est pointé et créé par le geste maternel, c'est donc la place où "pourra" se loger le père (á entendre comme fonction, comme Autre de la mère) pour qu'elle puisse borner sa propre toute-puissance en révélant son manque. La place du père se crée donc avant que son nom se ne prononce. C'est ainsi aà l'occasion d'une parole régie par l'hypothétique ${ }^{25}$ - c'est-à-dire relevant à la fois d'une anticipation et d'une anticipation supposant l'acceptation du manque ${ }^{26}$ : Tout mais pas ça!n (p. 261) - que cet acte de parole maternel (performatif s'il

24 Jean Bergès, *Clinique de l'hypothèse *, Texte, LImaginaire de la thérorie, op. cit., p. 260.

25 Et "[q]ue le nom du père ne puisse être avancé que dans l'hypothétique, voilà qui nous est familier ", nous rappelle Bergès ( ibid.).

26 Bergès pose alors cette question: «Dans quel entonnoir, vers quelle bouche aspirante est lancée comme un vecteur aux armes du désir cette anticipation? Vers ce que l'on appelle avec Lacan "le Grand Autre". Dans la mesure où il comporte un trou." (p. 261). Ainsi le Tout mais pas fa! du Dieu cle la Genèse en ferait-il un sujet aux prises avec l'émergence dans son discours de \$2, c'est-à-dire de l'Autre: "À savoir que [son] hypothèse n'est concevable que de se soutenir de la possibilité de substituer un signifiant à un autre " (ibid., p. 260). Tout mais pas ça fonde le désir de Dieu comme désir de l'Autre auquel se soumettent et auront à répondrent tous les sujets humains. Il n'y a donc pas de niveau métasymbolique où résiderait Dieu, pas d'avant puisque l'interdit, le désir de l'Autre, est là de tout temps. Pas d'Autre de l'Autre. Pas cle sortie possible sinon dans les "solutions oniriques". dans les croyances leurrantes qui empêchent le sujet, l'obligeant à répéter les mêmes scénarios, à se complaire clans les mêmes souffrances. 
114

en est puisqu'il invente la place du père) marquera son désir en énonçant un: Je ne suis pas satisfaite, il me manque Ça. Déphallicisation de la mère bénéfique donc, puisqu'elle ouvre un espace de liberté au sujet qui peut commencer à penser, c'est-à-dire à faire des hypothèses sur le lien de l'énonciatrice avec ce $C a$ mystérieux. En effet, "[s]i je désigne quelque chose par un geste du cloigt, on ne saura jamais si mon doigt désigne la couleur de l'objet ou sa matière, ou si c'est une tache ou une fêlure, etc. Il faut le mot, le discours pour le discerner. Il y a une propriété originale du discours par rapport à l'indication "27. C'est que le geste ostensif crée le lieu tiers, "met sur la piste de l'objet métonymique", c'est-à-dire de l'objet de désir (par opposition à l'ohjet mythique) en ce que ale regard de l'enfant se détache du désir de la mère [de ce que la mère veut de l'enfant] pour créer son objet dans l'autre" 28 . Cette mère qui parle passe dans la dimension de l'avoir (ou pas) alors qu'elle risquait, sans cette restriction anticipatrice, de rester dans le lieu sans faille et sans manque de l'être, sans ek-sistence (dans le sens heideggerien repris par Lacan), et de condamner son enfant à la fascination sidérée du rapport incestueux.

Savoir, c'est bien perdre le paradis, c'est réaliser, à partir d'une position de déficience, que d'objet de besoin, il n'y a "plus". Pourquoi? Parce que Je ne peux être satisfaisant pour Dieu-la-Mère - à entendre, rappelons-le, comme la première représentation d'une harmonie entre le sujet et le monde "inventée" a posteriori de sa perte. C'est que, dans le meilleur des cas, la mère désire ailleurs. Je ne suis donc pas tout pour elle. Je n'ai pas ce qu'il faut pour la satisfaire. C'est l'Autre qui l'a. Et elle désire ce qu'il a. L'arbitraire de ses va-et-vient s'explique: il existe une cause qui la transcende. Au-delà du premier Dieu, un autre Dieu? Parlé par la mère, qui a donc une place discursive (objet qui fonde le discours maternel comme celui d'un sujet désirant) avant de s'incarner. Le nom du père, c'est le nom sur les lèvres de la mère qui ne se prononce qu'à signifier au sujet son renvoi de sa place privilégiée. Sans cette chute de la mère, qui équivaut à la réalisation qu'elle est assujettie au désir (de l'Autre), le sujet

27 Jacques Lalan. Le seminaire, Libre III: Les psychoses, Paris, Seuil. coll. "Le Champ freudien", 1975, p. 155 (cité par C. Ferron: "Qu'est-ce que cest que (a? $\%$, p. 109).

28 Catherine Ferron, "Qu'est-ce que c'est que ça?-, op. cit., p. 109. 
ne pourra penser, imaginer l'objet comme telos possible des discours, ne pourra en fin de compte se situer comme Je et, de cette place universalisante, s'autoriser à désirer.

On dirait ainsi que le Dieu de la Genèse permet aux hommes de se séparer en leur laissant l'espace de liberté pour commettre la faute. La pomme, symbole de son savoir sur le désir - d'un désir situé donc au-delà du besoin - est l'objet métonymique premier, objet insaisissable puisque sa détention se paie du rejet hors du monde de la jouissance. Si vous en mangez, vous deviendrez Dieu a bien compris le serpent qui l'instaure ainsi comme désirable mais aussi inaccessible. Par cette parole (tentatrice), devenue discours - un discours qui se constitue par et autour de l'objet - le paradis devient un espace transitionnel, dirait Winnicott, une aire de jeu sur laquelle devra régner un autre Dieu, non plus figure maternelle mais paternelle, énonciateur des règles, des Tables de la Loi.

\section{La fin de l'histoire: composer avec l'impossible 29}

Dans le mythe biblique, Dieu apparaît à la fois comme la bonne marraine, qui donne sans compter mais qui escompte bonté et reconnaissance, et comme la marâtre injuste et arbitraire qui impose épreuve après épreuve pour tester son pouvoir, sa malédiction annonçant douleur et frustration, mais également une advenue certaine sinon au désir, du moins à un vouloir individuel. À la fin du conte, la souillon Cendrillon a passé l'épreuve de la castration imposée par la rivalité de la "femme de son père" en étant salie, avilie, c'est-à-dire déplacée de sa position de "fille préférée", toute satisfaisante. Ces épreuves traversées par les héros et les héroïnes des contes élaborent symboliquement les effets des castrations symboligènes - qui sont les conditions de l'acquisition du savoir - chez les enfants: elles racontent en fait comment le symbole peut servir de compensation aux pertes successives, aux chutes incessantes subies par le sujet hors du paradis de l'inceste. Les contes sont des métaphorisations des solutions oniriques qui servent, quant à elles, au niveau primaire, à éviter les coups au narcissisme (dit "primaire"). Ils proposent, au niveau secondaire, des théories de l'cedipe (pas toujours édul-

29 Expression cle Joyce McDougall; voir plus loin. 
corées, si nous pensons à Peau d'âne), et montrent comment les épreuves de séparation mènent à une autre sorte de jouissance tout aussi bonne. "On la mena chez le jeune prince, parée comme elle l'était: il la trouva encore plus belle que jamais, et peu de jours après, il l'épousa." ${ }^{30}$. À la fin, le prince est là comme prix pour garantir que le compte est bon, que l'on y retrouve ses billes. Comme partenaire appartenant à l'autre sexe mais à la même génération, il est, en tant que "fils du roi", le futur héritier du sceptre. Comme valeur sûre, il permet la sortie du monde injuste géré par la marâtre, de la même façon que l'arrivée de celle-ci avait éjecté la jeune fille de sa place rose-bonheur entre la "vraie" mère aimante et le père comblé.

Mais disons quand même avec Charles Melman que cette insistance sur la beauté de l'héroine est un peu louche, car "Il]al beauté [n']est[-elle pas] la tentative ultime de maintenir une présence qui, quoique phallique, protège du désir. Intimidante, elle est bien... rempart ultime contre la mort a moins que la révélation soudaine d'une faille, du défaut minuscule fasse basculer brutalement dans la fosse d'Eros [...]"31. Nul' "danger" de cela dans le conte de fée. C'est par cette beauté quasi mortifère que le conte représente le bonheur final comme s'accomplissant dans l'éternité du rapport sexuel, dans la parfaite complémentarité du masculin et du féminin. Le monde qui avait été troublé par le manque du héros, par sa frustration issue de l'usurpation de sa place d'enfant magique (comblant sa mère et son père), redevient présymbolique, si l'on peut dire. Le temps s'arrête, l'éternité se réinstalle. Sans événement, sans césure et donc sans temporalité, le symbolique s'abolit, le "sujet" s'efface à l'horizon de son bienêtre.

Le désir de jouissance du sujet humain se fonde de ce regret que représentent le mythe du paradis et le monde des fées: celui d'un monde magique où la chose est convocable par son signe, où le désirant et le désirable peuvent faire Un, où l'homme et la femme sont complémentaires. La croyance magique, toujours disponible, rappelle que le désir est désir d'une jouissance toujours incestueuse puisqu'il travaille à "retrouver" un monde où l'arbitraire du signifiant et la Loi de la prohibition de l'inceste n'auraient pas encore frustré le sujet, ne lui auraient pas encore signi-

30 Charles lerreault, upeau d'âne. Comtes, sp. cit. p. 283.

31 Charles Melman. Nontelles études sur lbysterie. op. cil. p. 142-143. 
fié son impuissance, son absence à lui-même, son aliénation au symbolique qui ne le représente que par intermittence et toujours comme manque. Le monde de la fin des contes, atemporel, est bien une solution onirique pour pallier l'angoisse d'un sujet jeté, dès son entrée dans le monde, dans l'arène des désirs des autres, sur la carte déjà tracée d'un symbolique où l'attendent les places universelles du "Je" et du "Tu*. Unique en tant qu'objet du désir de ses parents, mais membre de l'espèce humaine en tant que parlé et parlant, le sujet devra s'approprier le langage et ses formes sensibles pour tenter de se saisir de l'insaisissable objet. Par là il pourra fonder son je-ici-maintenant mortel et marqué par la différence sexuelle, comme le lieu d'une origine unique mais transformable en une histoire communicable aux autres. Car

[...] pour parvenir à composer avec l'impossible, l'individu n'est pas seulement écartelé entre le rêve et le délire. Une troisième scène lui est offerte dans l'espace qui sépare son monde interne de la réalité. Je veux parler de ce que Winnicott a appelé l'espace transitionnel. Ce lieu, défini, d'un côté, par la satisfaction pulsionnelle complète et, de l'autre, par l'objet à jamais inatteignable, est l'aire où se déploient tous les champs de la culture; une sorte d'espace-temps où se dépense l'essentiel cles forces humaines. Or, pour certains Je, cette aire de la culture est fort réduite, voire ignorée et inexplorée. Pour d'autres, elle est remplie d'objets transitionnels que nous pourrions considérer comme pathologiques ou, pour mieux dire, d'objets transito $i$ res. / Pensons ici à l'objet toxicomaniaque des addictions [...]. ${ }^{32}$

Ne pas (savoir) lire, c'est se refuser la seule porte de sortie du sujet qui est *là " par la force du symbolique et qui ne pourra en sortir que par la force de ce même symbolique. L'enfant non lecteur s'étant pourvu d'une croyance qui fait système hermétique, l'espace pour l'hypothèse et le doute, pour la conjecture et la pensée est réduit à une peau de chagrin qui l'opprime et le satisfait en même temps. Telle est la difficulté :

L'enfant non lecteur ne peut pas consentir au sacrifice, à la reconnaissance du corps de sa mère comme séparé du sien. Il reste dans une extériorité intérieure, un dehors-dedans du rapport sexuel fantasmatique, une dénégation. Le travail de l'analyse est d'aider au refoulement, à vectoriser l'intention car consentir au sacrifice du 1 c'est se compter, se détacher de la mère. ${ }^{33}$

32 Joyce McDougall, Théâtres du Je, op. cit., p. 13-14.

33 Catherine Ferron, *Enfants non lecteurs en thérapie ", op. cit., p. 264. 
118

Des épreuves de castration et de séparation, les enfants non lecteurs ne veulent rien savoir. On peut maintenant le dire autrement: c'est de ce qu'ils ne veulent rien (en) savoir, que certains enfants "réussissent" à ne pas apprendre à lire. Le sacrifice n'aura pas eu lieu qui apaise le regard de l'Autre: l'appel à la pensée magique en sera la conséquence car elle permet de continuer à croire à la création sui generis de l'objet de jouissance. Croire au Grand Livre magique, c'est attendre le retour du paradis perdu, le coup de baguette qui leur révèlera, en un éclair, le sens des mots/le sens des choses (les deux étant en miroir grâce à la toute-puissance de la *langue maternelle". Entre temps, en attendant, ils évitent de se confronter à la signification - c'est-à-dire à la différence et à l'arbitraire du code, du symbolique - en ne lisant pas, de peur de savoir ce que l'on veut d'eux, ce qu'ils veulent dire comme fils ou fille dans le désir de leurs parents. Ils restent ainsi attachés à ce paradis d'avant la chute, où tout est "motivé", en repoussant celle-ci vers un futur mythique (et non pas hypothétique. Le mode conditionnel du mythe - qui est aussi celui des enfants qui jouent: "Tu serais un prince et moi, la princesse, d'accord?" - marque leurs énoncés d'un virtuel impossible: Un jour, je saurai(s) lire... disent-ils. Quand? Quand je serai(s) grand. C'est-à-dire? Quand j'aurai(s) ce qu'il faut pour protéger/satisfaire Maman. Comme Papa (qui deurait l'avoir) ${ }^{34}$.

Ainsi pour ne pas commencer à se raconter leur histoire, ne pas se donner les moyens de la lire dans des livres écrits par d'autres, les non-lecteurs sont des croyants : ils font un acte de foi en gardant Dieu à sa place et en vénérant ses reliques: des lettres-images qui vont seulement par leur nom, dans cette épellation qu'ils pensent être la lecture, des mots-choses qui appartiennent au monde des fées et qui ne disent rien qui vaille sinon sous le regard de la mère qu'ils ne peuvent quitter. Leur dieu est un dieu lecteur: il a lu et il connaît le sens de leur vie car c'est leur destin qui est écrit dans son Livre.

\section{**}

34 C'est ce rapport au adésir du père" et du jeu qu'il instaure (ou pas) clans la alangue maternelle qu'il nous faudra travailler plus amplement dans un travail ultérieur. 
Qu'adviendra-t-il de la petite Catherine? Est-ce en retournant au Portugal qu'elle pourra s'autoriser à "chuter " hors de la nostalgie qui l'attache au pays mythique de sa naissance? Pourra-t-elle, dans sa psychothérapie, être confrontée à la place mortifère qui lui est allouée dans le désir d'enfant de la mère, entre deux morts? Deviendra-t-elle "coiffeuse comme maman avant" pour (se) faire (la) belle? Et rester Belle au Bois dormant "pour papa"? Ou pourra-t-elle courir le risque de ne plus être "tout pour papa", ce pauvre papa qui ne sait pas non plus, castré et aimé pour cela? La recherche de la beauté, visible dans ses *arrangements avec les objets", pourra-t-elle alors être dérangée pour laisser la place à un autre ordre? Celui de la différenciation symbolique et des lois de la connaissance? Apprendra-t-elle enfin à se lire sans faire appel au regard de l'Autre? Sans le Grand Livre du Monsieur portugais qui en savait plus que son père? 